BMJ Open

Sport \&

Exercise

Medicine

\title{
Effect of a personalised mHealth home-based training application on physical activity levels during and after centre-based cardiac rehabilitation: rationale and design of the Cardiac RehApp randomised control trial
}

Thijs Vonk (D) , ${ }^{1}$ Esmee A Bakker, ${ }^{1}$ Erwin S Zegers, ${ }^{2}$ Maria T E Hopman, ${ }^{1}$ Thijs M H Eijsvogels (D) ${ }^{1}$

To cite: Vonk T, Bakker EA, Zegers ES, et al. Effect of a personalised mHealth home-based training application on physical activity levels during and after centrebased cardiac rehabilitation: rationale and design of the Cardiac RehApp randomised control trial. BMJ Open Sport \& Exercise Medicine 2021;7:e001159. doi:10.1136/ bmjsem-2021-001159

Accepted 17 August 2021

A Check for updates

(c) Author(s) (or their employer(s)) 2021. Re-use permitted under CC BY-NC. No commercial re-use. See rights and permissions. Published by BMJ.

${ }^{1}$ Department of Physiology, Radboudumc, Nijmegen, The Netherlands

${ }^{2}$ Department of Cardiology, Canisius Wilhelmina Ziekenhuis, Nijmegen, The Netherlands

Correspondence to Dr Thijs M H Eijsvogels; thijs.eijsvogels@radboudumc.nl

\section{ABSTRACT}

Many patients lapse into a physically inactive lifestyle within months after cardiac rehabilitation (CR) programme completion. A mobile-health (mHealth) home-based training application can be used to intensify and/or prolong the CR programme to induce long-lasting improvements of habitual physical activity levels. This study will assess the effect of an additional home-based training module during CR and post-CR on habitual physical activity levels among coronary artery disease patients. A total of 132 patients ( $>18$ years old) will be recruited in an 18-week randomised controlled trial with four arms:

(1) 6 weeks centre-based CR (ie, standard care), (2) 6 weeks combined centre-based +mHealth home-based CR, (3) 6 weeks centre-based CR followed by 12 weeks mHealth home-based CR, (4) 6 weeks combined centrebased + mHealth home-based CR followed by 12 weeks mHealth home-based CR. The intervention groups will receive a daily and personalised exercise training using a smartphone application (Virtual Training) in addition to and/or as extension of the centre-based CR programme. The participants will be assessed prior to the centrebased CR programme, after completion of the 6 -week CR programme and after the 12 weeks extension. Primary outcome will be objectively measured habitual physical activity levels expressed as moderate to vigorous intensity activities (min/week). Secondary outcome parameters include sedentary behaviour, physical fitness (estimated $\mathrm{VO}_{2}$ max), handgrip strength, cardiovascular risk profile, quality of life and cardiac anxiety scores. The findings of the Cardiac RehApp study will provide insight into the added value of a personalised mHealth home-based training application on physical activity levels during and after centre-based CR. Trial registration number: NL72182.091.019.

\section{INTRODUCTION}

Cardiovascular diseases (CVDs) are the most common cause of mortality worldwide, responsible for almost one third of all deaths. ${ }^{1}$

\section{Key messages}

What is already known

- Home-based cardiac rehabilitation (CR) programmes are equally effective compared with centre-based CR programmes and may benefit from technological solutions such as mHealth or eHealth to deliver exercise training.

- Physical activity levels tend to decrease after completion of centre-based CR, but the effect size is limited and many patients relapse into a sedentary lifestyle within months.

- More intense training programmes and prolonged training programmes are known to induce larger and more sustainable improvements of physical fitness and physical activity among patients with cardiovascular disease participating in CR programmes.

What are the new findings

- The Cardiac RehApp study provides detailed insight into the acute and long-lasting effects of objectively measured physical activity and sedentary behaviour following four different CR programmes.

- A direct comparison of an intensified hybrid CR programme (centre-based +home-based) versus prolonged CR programme (home-based CR following traditional centre-based $\mathrm{CR}$ ) versus the combination of these CR programmes (ie, hybrid and prolonged) provides important information on which CR components induce the largest and most sustainable improvements in physical activity levels.

- The Cardiac RehApp study has an extensive measurement protocol allowing detailed phenotyping of CR-induced changes in cardiovascular risk factors across different domains (ie, behaviour, biomarkers, fitness, mental health).

The development of medical technology and therapeutic procedures have increased the rate of survival from acute and chronic cardiac conditions significantly. To prevent 
recurrence and to induce lower mortality rates, patients are advised to participate in a risk factor-modification programme such as cardiac rehabilitation (CR).

One of the cornerstones of CR is a (supervised) exercise training programme that aims to establish boundaries during exercise, to increase physical activity (PA) and fitness and to combat cardiac anxiety. ${ }^{23}$ Unfortunately, a large majority of patients with coronary artery disease (CAD) do not achieve the healthy lifestyle and therapeutic targets set by international health guidelines. ${ }^{4}$ Post-CR changes in habitual PA are small to modest and most patients relapse to an inactive lifestyle within months after completion of CR. ${ }^{5}$ The limited number of training sessions (ie, 12 session) during CR and the lack of guidance post-CR likely contribute to these disappointing outcomes. ${ }^{6}$

A potential solution to improve habitual exercise volumes is home-based CR. ${ }^{7}$ Previous studies reported that home-based CR is equally effective as centre-based CR in improving clinical and health-related quality of life (QoL) outcomes. Moreover, recent evidence suggests that the combination of home-based CR with centrebased CR might be superior in participant adherence. ${ }^{8}$ Previous studies investigating this form of CR often assessed physical fitness $\left(\mathrm{VO}_{2} \max \right)$ as their primary outcome on effectiveness. Although cardiorespiratory fitness is an important indication of overall physical health, objectively measured PA levels and sedentary behaviour (SB) are also strong predictors for health outcomes and provide important additional information on the effectiveness of CR programmes. However, the evidence on objectively measured PA levels and SB during and after different forms of CR is predominantly based on quasi-experimental or observational studies. ${ }^{9}$ Therefore, larger well-designed randomised clinical trials are warranted that include objectively measured PA and SB levels combined with different forms of CR.

The primary aim of this study is to investigate the effect of a home-based training module using a mHealth application added during CR and/or post-CR on habitual PA levels expressed as time spent on moderate to vigorous intensity PA (min/week). Secondary aims of this randomised controlled trial are to investigate the effects on $\mathrm{SB}$, physical fitness (estimated $\mathrm{VO}_{2} \max$ ), handgrip strength, cardiovascular (CV) risk profile, quality of life and cardiac anxiety scores. We hypothesise that using a combined programme of centre-based CR and homebased personalised training results in a greater increase in PA levels, physical fitness, handgrip strength, quality of life and a decreased total sitting time and cardiac anxiety score compared with usual care.

\section{METHODS}

\section{Study design}

In order to assess the effectiveness of a home-based personalised training module in addition to centre-based CR to improve habitual PA levels of CAD patients, an 18-week randomised controlled trial with four arms will

\begin{tabular}{ll}
\hline Table 1 & Treatment arms \\
\hline $\mathrm{CR}$ & 6 weeks of standard centre-based CR \\
& 12 weeks of usual care after completion centre- \\
& based CR \\
$\mathrm{CR}+$ & 6 weeks of standard centre-based CR \\
& 12 weeks home-based exercise training after \\
& completion centre-based CR \\
$+\mathrm{CR}$ & 6 weeks of standard centre-based CR combined \\
& with home-based exercise training \\
& 12 weeks of usual care after completion centre- \\
& based CR \\
$+\mathrm{CR}+$ & 6 weeks of standard centre-based CR combined \\
& with home-based exercise training \\
& 12 weeks home-based exercise training after \\
& completion centre-based CR
\end{tabular}

CR, cardiac rehabilitation.

be performed. Participants will be randomised into four groups as described in table 1 .

\section{Study setting and recruitment}

Participants will be recruited from a large hospital (Canisius Wilhelmina Ziekenhuis) in the south-eastern part of the Netherlands. The hospital offers an outpatient CR programme with a supervised exercise training programme of 6 weeks. Patients who are invited to participate in CR are screened for inclusion criteria and informed about the study by the specialised CR nurse. Hereafter, a detailed participant information sheet and informed consent form will be handed over. During the CR orientation visit, which is planned within 1-3 weeks after CAD diagnosis, eligible patients will be approached by a member of the research team. Additional information regarding the study will be provided and obligations of the participant will be clearly explained once more. If the patient is willing to participate in the study and deemed eligible, an informed consent form is signed. Figure 1 presents the timeline of participants during the study.

In order to be eligible to participate in this study, a subject need to meet all of the following inclusion criteria: participants must be (1) older than 18 years of age, (2) diagnosed with CAD (ST-elevation myocardial infarction-non-ST-elevation myocardial infarctionunstable angina pectoris-stable angina pectoris), (3) able to use a smartphone with the Virtual Training application, (4) referred to CR, (5) able to understand and perform the study procedures. Exclusion criteria are (1) being not in the possession of a smartphone, or being unable to operate a smartphone for the purpose of the trial (because of vision, hearing and cognitive or dexterity impairment), (2) having no (mobile) internet access at their place of residence, (3) having contraindications to perform exercise during the rehabilitation programme, (4) subjects who have severe orthopaedic problems that restricts PA, (5) having a significant language barrier. 


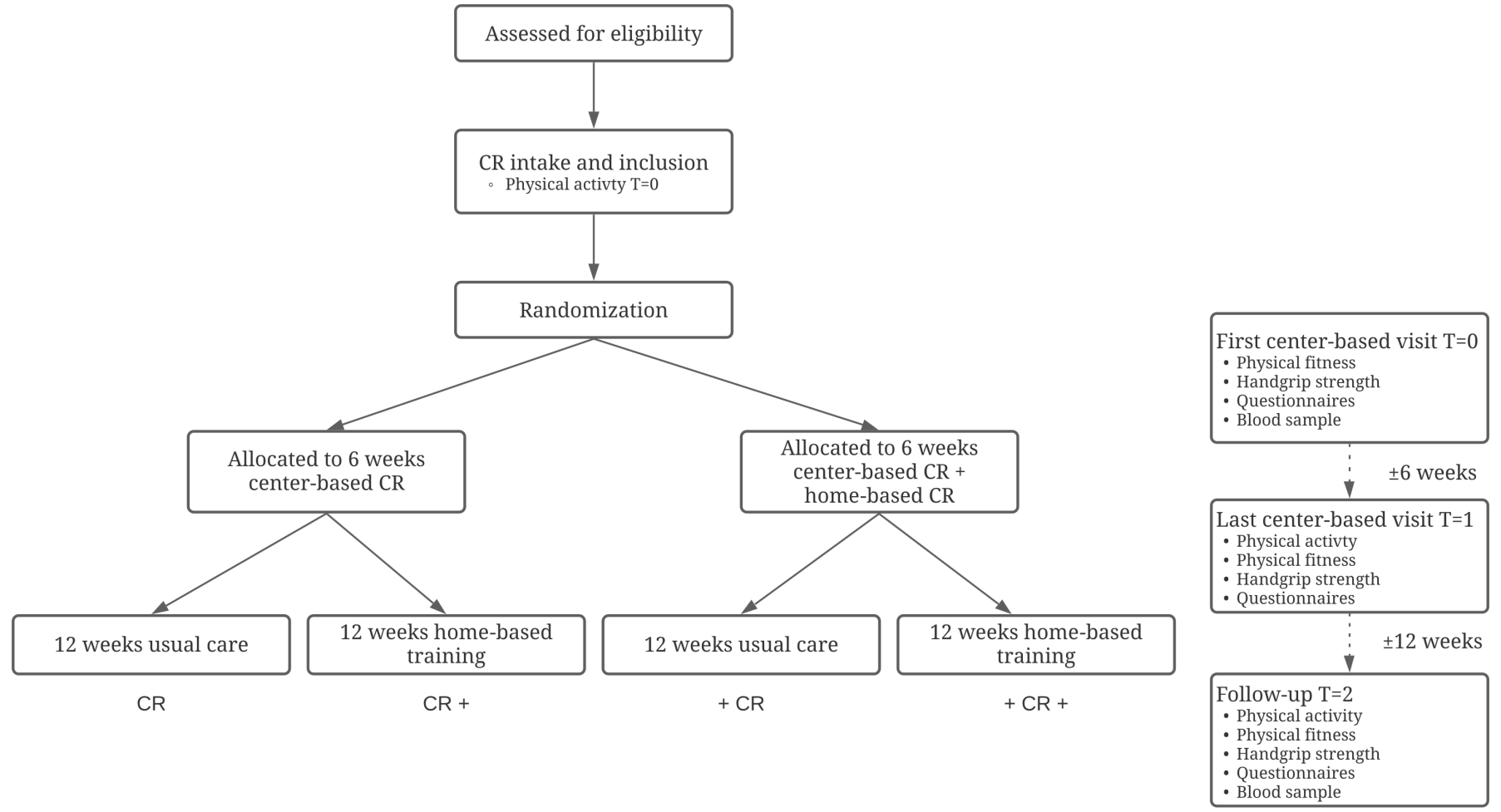

Figure 1 Flowchart, illustrating randomisation and measurements. CR, cardiac rehabilitation.

\section{Randomisation}

After signing informed consent, subjects will be randomly assigned to one of the four training intervention groups. We will use software (Castor EDC 2021, Ciwit B.V., Amsterdam, The Netherlands) with a variable block randomisation to allocate the included patients to one of the four treatment arms, stratified by index diagnosis (acute event vs elective procedure) to ensure all groups have equal amounts of CVD type. There is no blinding after randomisation due to the nature of the intervention, making it impossible to blind the participants or the research team.

\section{Usual care}

All patients will receive usual care and will be seen by their cardiologist as clinically appropriate. Usual care consists of a 6-week comprehensive CR programme. Before, during and after the CR programme, regular individual consultations are scheduled with the CR nurse focussing on lifestyle improvement, medication adherence and psychosocial well-being. The nurses contributing to the secondary prevention programme are registered nurses with experience in the care of cardiac patients. Prior to participation in the CR programme, all patients undergo a bicycle cardiac stress test to assess exercise tolerance and detect abnormalities followed by an appointment with their cardiologist. Within the usual care CR programme, group sessions are focused on exercise under the supervision of a physiotherapist. These group sessions of approximately 1 hour take place two times a week for a duration of 6 weeks. If indicated, the standard CR programme can be extended by a dietary module, psycho-educative prevention module and psychological module or by additional consultations with the CR nurse.

\section{Home-based program}

Subjects in the home-based groups CR+, +CRand +CR+ will be instructed to perform daily physical activities in their home situation using the Virtual Training mHealth smartphone application (Welfaster ApS, Esbjerg, Denmark) (figure 2). The application is able to show different training programmes including both strength and aerobic exercises, such as squats, walking, running and provides instructions in the form of both video, text and audio during the exercises. At baseline, the researcher will guide the participant to set goals based on preferences and physical status, and subsequently construct a personalised home-based training programme. Goal setting is based on exercise preference (ie, biking more enjoyable then walking) and physical limits based on previous exercise experience, age and possible orthopaedic limitations. The training programme consists of a combination of aerobic and resistance exercises. Progression regarding intensity and feasibility of the exercises will be monitored with a weekly text message in the application. A reminder will be sent automatically after missing three subsequent training days. Patients can also contact the research team by sending an in-application message in the Virtual Training application. Aerobic exercises consist of an incremental walking, biking or running training programme with the goal to increase daily PA duration. Patients are encouraged to exercise daily, starting at a low intensity and duration and gradually increase duration and distance. Aerobic exercises 
A

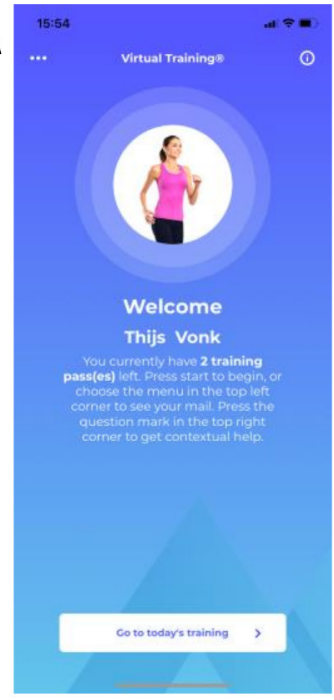

D

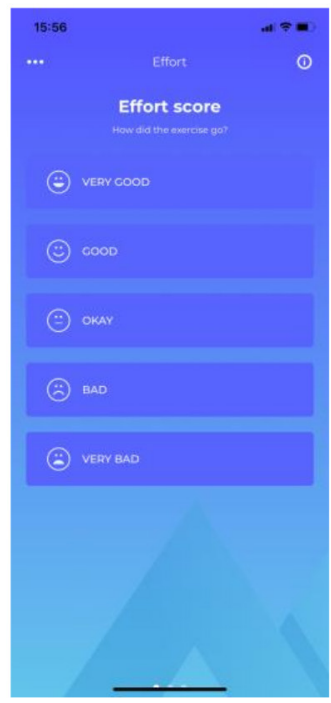

B

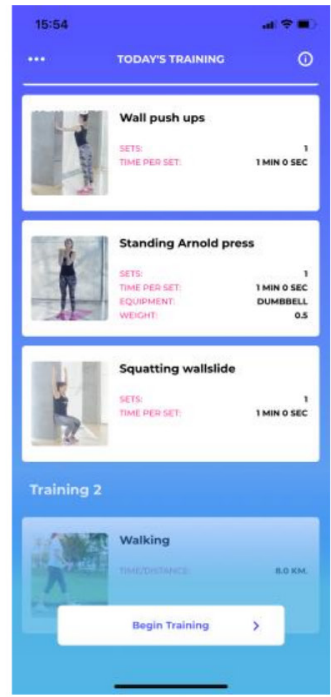

E

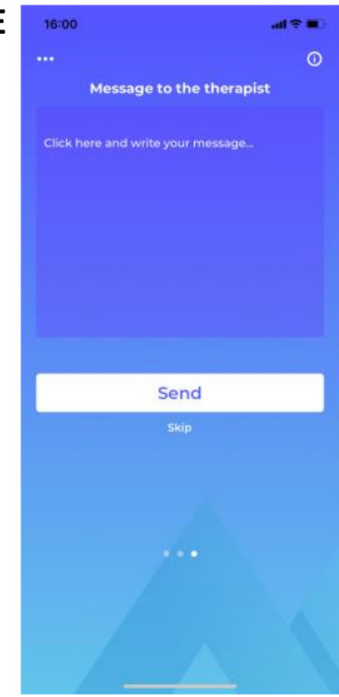

C

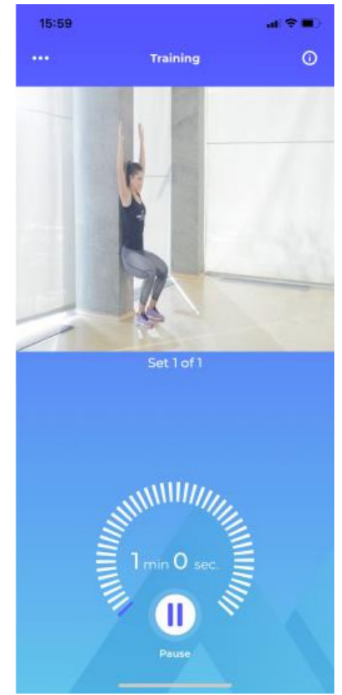

$\mathbf{F}$

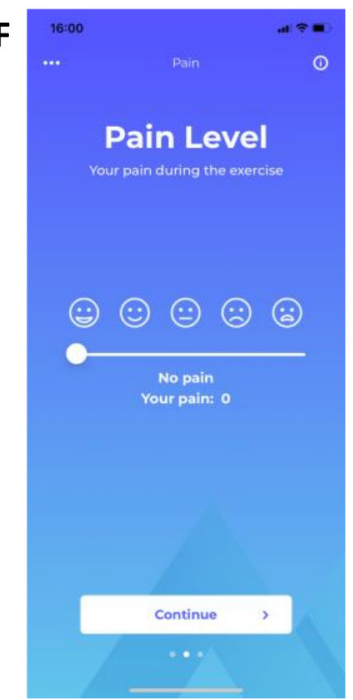

Figure 2 Example of the front-end of the virtual training application.

start at $5-10 \mathrm{~min}$ and gradually increase to $>30 \mathrm{~min}$ per day. Resistance exercises consist of 5-10 bodyweight exercises focussing on both upper and lower body which patients can perform in their home environment. Exercises are performed in 1-3 sets consisting of either 10-15 repetitions or are timebound (ranging from 30 to $120 \mathrm{~s})$. Subjects will be instructed to perform the bodyweight exercises at least two times a week on a self-chosen moment using the Virtual Training application. During the centre-based rehabilitation period (weeks 1-6), the intensity of the home-based exercises will be adjusted to the patient's physical status and level of PA. In the beginning, the strength exercise programme will consist of lighter exercises and longer breaks. Compliance will be monitored via the Virtual Training application and patients receive a weekly text message to ask about the intensity of the programme and whether it is needed to make adjustments to the training schedule. Based on the individual participants progression, the intensity of exercises and total training programme will be increased over time.

\section{Outcome measures}

The primary outcome of the Cardiac RehApp study will be objectively measured habitual PA levels expressed in time spent on moderate to vigorous intensity activities (min/week). Secondary outcome parameters include total sitting time ( $\mathrm{min} /$ week), physical fitness (estimated $\mathrm{VO}_{2} \max$ ), handgrip strength, $\mathrm{CV}$ risk profile, quality of life and cardiac anxiety scores. All participants will be assessed during three different timepoints: at baseline (prior to the CR programme $\mathrm{T}=0$ ), after completion of the 6 -week CR programme $(\mathrm{T}=1)$ and after 12 weeks of CR extension or usual care (ie, self-guidance, $\mathrm{T}=2$ ) (figure 1). Outcome measures will be conducted at the same hospital. 


\section{Habitual PA}

PA patterns will be objectively measured with a validated accelerometer (ActivPAL micro, PAL technologies, Glasgow, UK)..$^{10}$ The ActivPAL is a small device $(25 \times 45 \times 5 \mathrm{~mm})$, which is attached to the thigh by one of the researchers using hypoallergenic and transparent tape (Tegaderm, 3M), and collects data about static and dynamic acceleration as well as posture at a sampling rate of $20 \mathrm{~Hz}$. To allow for continuous monitoring, the ActivPAL is sealed with a nitrile sleeve and transparent tape for waterproof protection. The monitor is worn for eight consecutive days for 24 hours per day. During these measurements, patients are required to fill in a sleep diary. After wearing the ActivPAL for eight consecutive days, the ActivPAL will be send back to our institute by registered mail parcel. The raw data will be extracted using the PAL Software Suite V.8 and analysed by a modified version of the script of Winkler et al using sleep/ wake diaries. ${ }^{12}$ Sedentary time will be defined as any waking behaviour characterised by an energy expenditure $\leq 1.5 \mathrm{MET}^{13}$ while in a seated, reclined or lying posture. ${ }^{1415}$ The accumulation of sedentary time will be defined as short $(<5$ consecutive minutes $)$, medium (5-29 consecutive minutes) and prolonged ( $\geq 30$ consecutive minutes) sedentary bouts. Light intensity PA will be defined as standing time combined with stepping time with MET-values <3. Moderate-to-vigorous PA will be defined as stepping time with MET-values $\geq 3$. ${ }^{16}$

\section{Physical fitness}

A submaximal cycling test will be performed to examine physical fitness, expressed as estimated maximal oxygen consumption. The Astrand-Rhyming test is a frequently used single-stage cycle ergometer test. ${ }^{17}$ In short, subjects perform a 6 -min cycling test, preceded by a $4-5 \mathrm{~min}$ warm-up phase. After the 4-5 min warming-up period patients cycle for $6 \mathrm{~min}$ on a stable workload while heartrate is monitored continuously and the Borg score is noted during the 3rd and 6th minute. After warming-up, workload is increased to ensure that heart rate maintains at a stable level between 110 and 140 beats/ min during the 5 th and 6 th-min of cycling test. Based on gender, workload, weight and heart rate of minute 5 and 6 , maximal oxygen consumption is estimated. Heart rate will be monitored continuously during testing by a Polar heart rate monitor (Polar V800, Kempele, Finland). Patients using betablocker medication follow an adjusted test in which the workload is steadily increased similar to the standard test until a Borg Rating of Perceived Exertion score of at least 12 is reached.

\section{Handgrip strength}

Muscle strength will be measured by handgrip strength of the dominant hand. This will be assessed with a hydraulic, analogue hand dynamometer (Jamar, Jackson, Michigan, USA) adjusted to the hand size of every patient. The patient is seated in a chair with the elbow flexed in a 90 degree angle position. Arm support by the chair is not allowed. Three measurements will be performed with approximately $30 \mathrm{~s}$ rest between measurements. The maximum strength effort in kilograms will be used for analysis.

\section{Quality of life and cardiac anxiety}

Quality of life will be measured using the HeartQoL. ${ }^{18}$ This is a health-related quality of life questionnaire and has been found to be both valid and reliable in patients referred to CR. Cardiac anxiety/personality will be measured using the Cardiac Anxiety Questionnaire. ${ }^{19}$ Online questionnaires will be sent to participants by email and take approximately $20 \mathrm{~min}$ to complete.

\section{Blood samples}

A venous blood sample (obtained by venepuncture) is collected to assess low-density lipoprotein cholesterol, high-density lipoprotein cholesterol, total cholesterol, triglycerides, glucose and haemoglobin at baseline and at 18 weeks of follow-up.

\section{Data management and accessibility}

All data will be stored in the Digital Research Environment (www.andrea-consortium.org), an online and easy-to-use cloud platform which allows users across the globe to merge, analyse, store, share, archive and publish data in a safe and compliant way (ie, security, information and communications technology (ICT) infrastructure, audit trail, general data protection regulation (GDPR) compliant). Standard software packages are available for data handling and statistical analyses (eg, SPSS, R, SAS). In line with the Open Science initiative and the FAIR principles (Findable, Accessible, Interoperable and Reusable), data from the Cardiac RehApp study will be available for reuse on reasonable request via the corresponding author.

\section{Sample size}

Sample size was calculated using G*power (V.3.1.9.4, Kiel University, Germany). A power calculation was performed with differences in PA volumes (expressed in $\mathrm{min} /$ week) as the primary outcome measure. The sample size (n) was calculated with a power of $80 \%(1-$ $\beta=0.80)$ and a significance level of $5 \%(\alpha=0.05)$. We used data from a previously published study as a reference that looked at changes in PA before and after CR using objective measurements. ${ }^{12}$ They found a change in PA volume from pre-CR to post-CR of $0.65 \% \pm 2.21 \%$ of total daily wearing time during awake hours $(852 \mathrm{~min})$. This equals an increase of $7 \mathrm{~min} /$ day. According to these data, during CR an average increase of $49 \mathrm{~min} /$ week of moderate to vigorous PA was observed. In this study, the greatest difference is expected between the CR group (usual care) and the +CR+ group (home-based during CR and postCR). Several large-scale epidemiology studies have been performed that examined the dose-response relationship between PA and CVD and premature mortality. ${ }^{16-19}$ Small increases in exercise volumes as little as $10 \mathrm{~min} /$ day of brisk walking is associated with $33 \%$ risk reduction for 
all-cause mortality, whereas the greatest risk reductions occurs at levels of $150 \mathrm{~min}$ /week of moderate-intensity activity and is therefore recommended globally. ${ }^{20-23}$ Based on these previously reported risk reductions and recommendations, we believe an average difference of 20 min of moderate to vigorous PA per day in the $+\mathrm{CR}+$ group compared with the control group is a reasonable effect and is likely to have significant clinical relevance. Using the average standard deviation (23 min/day) measured in the previously mentioned study and the expected maximum difference between the four groups, an effect size of 0.33 was calculated. This resulted in a total number of participants in of 104. Taking a dropout of $20 \%$ into consideration, we will include $n=33$ participants in each group. With 33 participants in each group, the total calculated sample size is 132 .

\section{Statistical analysis}

Statistical analyses will be performed using SPSS V.25.0 statistical software (IBM, Chicago, Illinois, USA). Collected data will be checked for completeness and normality of distribution (checked visually and using the Kolmogorov-Smirnov test). All normally distributed data will be presented as means $\pm \mathrm{SD}$ or as median (IQR) when data are not normally distributed. Statistical significance will be set at $\mathrm{p}<0.05$.

Intention-to-treat analysis will be used, based on the group they were initially (and randomly) allocated to, regardless of whether or not they dropped out or fully adhered to the treatment. Summary descriptive statistics, including demographics, will be generated for baseline characteristics. Depending on distribution, baseline characteristics between the two groups will be compared using an independent student t-test or Mann-Whitney U test. Changes in time spent on moderate to vigorous PA measured by the ActivPAL will be used as primary study outcome. We will conduct linear mixed models to simultaneously investigate the effects of the CR interventions and how they change over time, and to investigate the effects of the CR intervention at each time point. To do so, we will use the interventions, time points and the interaction between the intervention and time points as categorical factors. When necessary, a random intercept will be added to the model. We will use a similar statistical approach to analyse differences in total sitting time, physical fitness and CV risk scores. Adherence to the centre-based CR programme and compliance to the Virtual Training programme will be reported as descriptive statistics.

\section{DISCUSSION}

The Cardiac RehApp study examines the effect of a personalised home-based mHealth intervention on objectively measured habitual PA levels during and after completion of centre-based CR. For this purpose, we will use a commercially available smartphone application which encompasses an extensive database with different types of aerobic and resistance exercises $( \pm 200)$ that can be prescribed to the individual patient. Due to the unique nature of our study design, using four parallel arms, the Cardiac RehApp study will provide insight into the independent effects of an intensified and prolonged centre-based CR with home-based exercise training, as well as the combination of these features. Hence, we are able to compare the benefits of three different mHealth interventions to usual care (ie, 6 weeks centre-based CR) on habitual PA levels.

Previous studies evaluating home-based CR found an equal effectiveness in improving clinical and healthrelated QoL outcomes compared with centre-based $\mathrm{CR}^{20}$ Most studies comparing clinical outcomes in patients participating in home-based or centre-based CR reported data on exercise capacity, often including gas exchange data (ie, $\mathrm{VO}_{2} \max$ ). The majority of these studies reported a similar improvement in cardiorespiratory fitness in patients following home-based compared with centre-based CR. ${ }^{21}{ }^{22}$ Cardiorespiratory fitness is an important indication of overall physical health, and while related, PA levels and sedentary time are both strong, yet different predictors for health outcomes. There has been an increase in evidence and emphasis of the importance of PA and SB by large organisations such as the WHO and American College of Sports Medicine. ${ }^{23}{ }^{24}$ Being physically active is associated with higher levels of functional health, a lower risk of falling, better cognitive function and has a strong dose-response relationship with cardiovascular outcomes. ${ }^{23}$ Therefore, it is vital to not only look at physical fitness, and include PA patterns as and outcome measure of effectiveness.

Despite the strong evidence and recommendations, only a few studies have looked at the effects on PA and SB levels during and after home-based or centre-based CR. ${ }^{25}$ Due to the novelty of the majority of the home-based CR studies, there is a large heterogeneity between the studies in type of programme (home-based, hybrid and centre-based), measurement of PA and/or SB (objective vs subjective measures) and intervention type (use of mobile application, video sessions, online training programme and so on). The heterogeneity across studies leads to a failing consensus of the effectiveness of different types of CR programmes on PA and SB levels during and after home-based and centre-based CR, and dedicated randomised clinical trials-such as the Cardiac RehApp study—are needed to clarify this issue. ${ }^{9}$

The form and follow-up period of exercise training programmes differ markedly across home-based and hybrid CR programmes, making it difficult to determine whether one approach is better compared with the another, and the overall impact of CR on objectively measures of PA and SB. In order to achieve sustainable behavioural changes, it is vital to motivate a patient and increase their self-efficacy and self-regulation in enhancing long-term PA. To promote this, a graded programme, supporting the transition from the centre to the home environment, may result in the best long-term results. ${ }^{26}$ Another benefit of the graded approach is that 
the exercise prescription can be personalised and adjusted during an early stage of the rehabilitation programme. This will lead to a training stimulus that matches the level of the patient, avoid over-training or undertraining and dropout due to injury or motivational problems that could omit the health benefits of the programme. We hypothesise that a hybrid programme in which the patient follows both centre-based CR supplemented with home-based training, graded into a personalised homebased only follow-up period may therefore be superior to other forms of CR as it includes the best of both worlds.

Standard centre-based CR programmes often offer a combination of machine-based strength exercises and aerobic training. However, the absence of adequate equipment in the home-environment often makes it challenging for patients to combine aerobic training with strength exercises. Undertaking strength training exercise is important because of the known positive effects on muscle mass and muscle function and is associated with a reduced risk of CVD and premature mortality. ${ }^{27}$ Furthermore, since muscle mass declines with age, undertaking muscle strengthening exercises is vital for combatting the negative effects of age-related decline in muscle mass. Therefore, it essential to incorporate strength exercises in home-based CR training programmes alongside aerobic training to ensure the optimal beneficial effects of PA, which is a unique advantage of our home-based mHealth programme.

\section{CONCLUSION}

The Cardiac RehApp study will evaluate the effect of a mHealth guided and personalised home-based exercise intervention during and after a traditional CR programme on objectively measured physical (in)activity patterns. Using a four-arm study design, the benefits of an intensified hybrid CR programme as well as extension of a traditional centre-based CR programme with homebased CR will be assessed. Beyond its impact on habitual $\mathrm{PA}$, we will also determine its effect on SB, cardiovascular risk factors, physical fitness, muscle strength, heartrelated quality of life and cardiac anxiety. Outcomes of the present study will contribute to optimise the content of CR programmes to induce long-lasting improvements in high-risk behaviour.

\section{Collaborators Not applicable.}

Contributors TV drafted the manuscript with input from TE and MTEH. All authors were involved in the critical revision of the paper for intellectual content and gave final approval before submission.

Funding This study is funded by The Eurostars Eureka Program.

Competing interests None declared.

Patient and public involvement Patients and/or the public were not involved in the design, or conduct, or reporting, or dissemination plans of this research.

Patient consent for publication Not required.

Ethics approval The study complies with the World Medical Association Declaration of Helsinki on ethics in medical research and is approved by the medical ethics committee of Arnhem/Nijmegen (NL72182.091.019). Netherlands
Trial Registration: NL9156. All participants are required to sign a written informed consent.

Provenance and peer review Not commissioned; externally peer reviewed.

Data availability statement Data are available upon reasonable request. All data will be stored in the Digital Research Environment (www.andrea-consortium.org), an online and easy-to-use cloud platform which allows users across the globe to merge, analyse, store, share, archive and publish data in a safe and compliant way (ie, security, ICT infrastructure, audit trail, GDPR compliant). Standard software packages are available for data handling and statistical analyses (eg, SPSS, R, SAS). In line with the Open Science initiative and the FAIR principles (Findable, Accessible, Interoperable and Reusable), data from the Cardiac RehApp study will be available for reuse upon reasonable request via the corresponding author.

Open access This is an open access article distributed in accordance with the Creative Commons Attribution Non Commercial (CC BY-NC 4.0) license, which permits others to distribute, remix, adapt, build upon this work non-commercially, and license their derivative works on different terms, provided the original work is properly cited, appropriate credit is given, any changes made indicated, and the use is non-commercial. See: http://creativecommons.org/licenses/by-nc/4.0/.

\section{ORCID iDs}

Thijs Vonk http://orcid.org/0000-0002-9822-834X

Thijs M H Eijsvogels http://orcid.org/0000-0003-0747-4471

\section{REFERENCES}

1 Cardiovascular diseases (CVDs) World Health organization, 2017. Available: www.who.int/en/news-room/fact-sheets/detail/ cardiovascular-diseases-(cvds)

2 Piepoli MF, Hoes AW, Agewall S, et al. 2016 European Guidelines on cardiovascular disease prevention in clinical practice: The Sixth Joint Task Force of the European Society of Cardiology and Other Societies on Cardiovascular Disease Prevention in Clinical Practice (constituted by representatives of 10 societies and by invited experts)Developed with the special contribution of the European Association for Cardiovascular Prevention \& Rehabilitation (EACPR). Eur Heart J 2016;37:2315-81.

3 Fihn SD, Gardin JM, Abrams J, et al. 2012 ACCF/AHA/ACP/AATS/ PCNA/SCAI/STS guideline for the diagnosis and management of patients with stable ischemic heart disease: a report of the American College of cardiology Foundation/American heart association Task force on practice guidelines, and the American College of physicians, American association for thoracic surgery, preventive cardiovascular nurses association, Society for cardiovascular angiography and interventions, and society of thoracic surgeons. Circulation 2012;126:e354-471.

4 Kotseva K, Wood D, De Bacquer D, et al. EUROASPIRE IV: a European Society of cardiology survey on the lifestyle, risk factor and therapeutic management of coronary patients from 24 European countries. Eur J Prev Cardiol 2016;23:636-48.

5 Bakker EA, van Bakel BMA, Aengevaeren WRM, et al. Sedentary behaviour in cardiovascular disease patients: risk group identification and the impact of cardiac rehabilitation. Int $J$ Cardiol 2021;326:194-201.

6 Ter Hoeve N, Sunamura M, van Geffen ME, et al. Changes in physical activity and sedentary behavior during cardiac rehabilitation. Arch Phys Med Rehabil 2017;98:2378-84.

7 Dalal HM, Zawada A, Jolly K, et al. Home based versus centre based cardiac rehabilitation: cochrane systematic review and metaanalysis. BMJ 2010;340:b5631.

8 Wu C, Li Y, Chen J. Hybrid versus traditional cardiac rehabilitation models: a systematic review and meta-analysis. Kardiol Pol 2018;76:1717-24.

9 Meiring RM, Tanimukai K, Bradnam L. The effect of Exercise-Based cardiac rehabilitation on objectively measured physical activity and sedentary behavior: a systematic review and meta-analysis. J Prim Care Community Health 2020;11:215013272093529.

10 Harrington DM, Welk GJ, Donnelly AE. Validation of Met estimates and step measurement using the ActivPAL physical activity logger. $J$ Sports Sci 2011;29:627-33.

11 Kozey-Keadle S, Libertine A, Lyden K, et al. Validation of wearable monitors for assessing sedentary behavior. Med Sci Sports Exerc 2011;43:1561-7.

12 Winkler EAH, Bodicoat DH, Healy GN, et al. Identifying adults' valid waking wear time by automated estimation in activPAL data collected with a $24 \mathrm{H}$ wear protocol. Physiol Meas 2016;37:1653-68.

13 Eckel RH, Jakicic JM, Ard JD, et al. 2013 AHA/ACC guideline on lifestyle management to reduce cardiovascular risk: a report of the 
American College of Cardiology/American heart association Task force on practice guidelines. Circulation 2014;129:S76-99.

14 Sedentary Behaviour Research Network. Letter to the editor: standardized use of the terms "sedentary" and "sedentary behaviours". Appl Physiol Nutr Metab 2012;37:540-2.

15 Tremblay MS, Aubert S, Barnes JD, et al. Sedentary Behavior Research Network (SBRN) - Terminology Consensus Project process and outcome. Int J Behav Nutr Phys Act 2017;14:75.

16 Lyden K, Keadle SK, Staudenmayer J, et al. The activPALTM accurately classifies activity intensity categories in healthy adults. Med Sci Sports Exerc 2017;49:1022-8.

17 Cink RE, Thomas TR. Validity of the Astrand-Ryhming nomogram for predicting maximal oxygen intake. Br J Sports Med 1981;15:182-5.

18 De Smedt D, Clays E, Höfer S, et al. Validity and reliability of the HeartQoL questionnaire in a large sample of stable coronary patients: the EUROASPIRE IV study of the European Society of cardiology. Eur J Prev Cardiol 2016;23:714-21.

19 van Beek MHCT, Voshaar RCO, van Deelen FM, et al. The cardiac anxiety questionnaire: cross-validation among cardiac inpatients. Int J Psychiatry Med 2012;43:349-64.

20 Frederix I, Caiani EG, Dendale P, et al. Esc e-Cardiology Working group position paper: overcoming challenges in digital health implementation in cardiovascular medicine. Eur J Prev Cardiol 2019;26:1166-77.
21 Kraal JJ, Peek N, Van den Akker-Van Marle ME, et al. Effects of home-based training with telemonitoring guidance in low to moderate risk patients entering cardiac rehabilitation: short-term results of the FIT@Home study. Eur J Prev Cardiol 2014;21:26-31.

22 Moholdt T, Bekken Vold M, Grimsmo J, et al. Home-Based aerobic interval training improves peak oxygen uptake equal to residential cardiac rehabilitation: a randomized, controlled trial. PLoS One 2012;7:e41199.

23 Bull FC, Al-Ansari SS, Biddle S, et al. World Health organization 2020 guidelines on physical activity and sedentary behaviour. Br J Sports Med 2020;54:1451-62.

24 King AC, Powell KE, Kraus WE. The US physical activity guidelines Advisory Committee Report-Introduction. Med Sci Sports Exerc 2019;51:1203-5.

25 Dibben GO, Dalal HM, Taylor RS, et al. Cardiac rehabilitation and physical activity: systematic review and meta-analysis. Heart 2018;104:1394-402.

26 Snoek JA, Eijsvogels TMH, VAN 't Hof AWJ, et al. Impact of a graded exercise program on $\mathrm{V}$ O2peak and survival in heart failure patients. Med Sci Sports Exerc 2018;50:2185-91.

27 Warburton DE, Gledhill N, Quinney A. Musculoskeletal fitness and health. Can J Appl Physiol 2001;26:217-37. 